\title{
Efficacy and Feasibility of Pain management and Patient Education for Physical Activity in Intermittent claudication (PrEPAID): protocol for a randomised controlled trial
}

\author{
Ukachukwu O. Abaraogu ${ }^{1,2^{*}}$ (D), Philippa M. Dall ${ }^{3}$, Julie Brittenden ${ }^{4,5+}$, Wesley Stuart ${ }^{4}$, Garry A. Tew ${ }^{6}$, \\ Jon Godwin ${ }^{7}$ and Christopher A. Seenan ${ }^{3+}$
}

\begin{abstract}
Background: Physical activity (PA) improves functional capacity and quality of life and provides secondary prevention benefits in individuals with peripheral arterial disease (PAD) and intermittent claudication (IC). However, pain and patient lack of knowledge are key barriers to the uptake of, and adherence to, PA recommendations. This trial will test the efficacy and feasibility of a non-invasive pain management intervention with and without patient education to improve PA in individuals with PAD and IC.

Methods: This is a randomised, controlled assessor-blinded feasibility trial with four parallel groups. Eighty adults with PAD and IC will be randomly assigned 1:1:1:1 to Active TENS (transcutaneous electrical nerve stimulation), Placebo TENS, Active TENS + Patient education or Placebo TENS + Patient education groups. All groups will continue to receive usual care over the intervention period. Participants randomised to Active TENS will receive a TENS device (preset at $120 \mathrm{~Hz}$, $200 \mu \mathrm{s})$ and will be instructed to use the device daily at home or elsewhere for 6 weeks with a patient-determined intensity of "strong but comfortable". Placebo TENS group participants will receive the same model of TENS device and instructions for use as those in the active group, except that the stimulation dose will be safely altered to produce nontherapeutic, ineffective stimulation. Participants randomised to patient education will receive a one-off 3-h workshop of structured group education (four to five persons in each group) and three sets of twice-weekly phone calls. Efficacy outcomes will be assessed at baseline, after 6 weeks of intervention and at 3 months follow-up. Absolute claudication distance using the Gardner treadmill protocol will be assessed as the primary outcome. Secondary outcomes will assess initial claudication distance, daily PA and patient-reported outcomes including quality of life, pain self-efficacy, depression, disease perception and walking impairment pain intensity and quality. Feasibility outcomes will assess rates of recruitment, retention and adherence. Focus groups with participants at the end of the trial will explore the acceptability of the interventions.
\end{abstract}

(Continued on next page)

\footnotetext{
* Correspondence: Ukachukwu.Abaraogu@gcu.ac.uk;

Ukachukwu.Abaraogu@unn.edu.ng

${ }^{\dagger}$ Julie Brittenden and Christopher A. Seenan contributed equally to this work.

${ }^{1}$ Centre for Living, School of Health and Life Sciences, Glasgow Caledonian

University, Glasgow, UK

${ }^{2}$ Department of Medical Rehabilitation, Faculty of Health Science and

Technology, College of Medicine, University of Nigeria, Enugu, Nigeria

Full list of author information is available at the end of the article
}

(c) The Author(s). 2019 Open Access This article is distributed under the terms of the Creative Commons Attribution 4.0 International License (http://creativecommons.org/licenses/by/4.0/), which permits unrestricted use, distribution, and

reproduction in any medium, provided you give appropriate credit to the original author(s) and the source, provide a link to the Creative Commons license, and indicate if changes were made. The Creative Commons Public Domain Dedication waiver (http://creativecommons.org/publicdomain/zero/1.0/) applies to the data made available in this article, unless otherwise stated. 
(Continued from previous page)

Discussion: This trial will determine the efficacy and feasibility of using a low-cost, CE-marked non-invasive pain management modality delivered with or without a patient-centred education intervention to improve PA in individuals with PAD and IC.

Trial registration: ClinicalTrials.gov, NCT03204825. Registered on 2 July 2017.

Keywords: Peripheral arterial disease, Transcutaneous electrical nerve stimulation, Exercise, Physical activity, Patientcentred care, Behavioural change therapy, Intermittent claudication

\section{Background}

Peripheral arterial disease (PAD) affects 2.7 million people in the UK [1]. The most common symptom that patients experience is intermittent claudication (IC), which is pain in the buttock, calf or thigh precipitated by exercise and relieved by rest [1]. The underlying cause of PAD is atherosclerosis, which leads to arterial stenosis and inadequate blood flow and tissue oxygen delivery during exercise [2-5]. Given the diffuse nature of atherosclerosis and the involvement of other arterial beds, patients with PAD and IC have a three to four times increased mortality compared to age and sex matched controls [6]. In addition IC has a major negative impact on patients' mobility and quality of life $[7,8]$.

Patients with symptomatic PAD should receive the same secondary prevention management as patients with symptomatic coronary artery disease [9]. Improving daily physical activity (PA) is particularly important in individuals with IC, as lower PA levels have been recognised as a strong predictor of increased morbidity and mortality in this population [10]. Current National Institute for Health and Care Excellence (NICE) guidelines recommend the use of supervised exercise programmes, encouraging patients "to exercise to the point of maximal pain", as first line treatment [11]. However, whilst supervised exercise programmes lead to a significant improvement in the absolute walking distances of patients with IC on a treadmill, it is unclear if this is sustained or leads to improvement in daily PA [12]. Furthermore, due to the resources required to deliver the recommended 3 -month exercise programme (30-45 min three times weekly), supervised exercise programmes are not always routinely available to UK National Health Service (NHS) patients. In addition, time and travel challenges (compounded by background mobility issues) including costs tend to lead to low patient uptake and high attrition rates [13]. Therefore, investigating the feasibility of using low-cost, patient-centred interventions that can support increased PA is warranted.

Lack of self-efficacy, attributed to poor understanding of the disease and uncertainty regarding the importance of exercise, has been shown to be a major barrier to exercise uptake in this population [14]. Also, for patients with IC to maximise the benefits of improved walking ability and secondary prevention, they are recommended to exercise beyond the point when pain occurs, which represents another barrier to engagement in PA [15]. These barriers to pain and lack of knowledge underscore the importance of including pain management and patient education components in a low-cost, patient-centred intervention as key to enhancing uptake and adherence to exercise recommendations in individuals with PAD/IC $[15,16]$.

Our group recently developed and piloted Structured EDucation for Rehabilitation in Intermittent Claudication (SEDRIC) [17], a patient-centred education intervention with the specific aim of educating patients with IC about their condition, improving patient ownership and promoting self-managed walking. We found that in patients with IC $(n=14)$, treadmill walking distances (30\%) and quality of life (32\%) improved from baseline after 6 weeks of structured education. In addition, there was a trend for patients to increase their daily PA (approximately $8 \%$ changes from baseline).

Similarly, we have demonstrated in an experimental lower limb ischaemic pain model in healthy volunteers $(n=28)$ that transcutaneous electrical nerve stimulation (TENS), a low-cost, CE-marked non-invasive pain management device, significantly increased pain threshold, tolerance and endurance compared to placebo TENS [18]. Our extension proof-of-concept pilot study demonstrated that TENS, when applied to patients with IC exercising on a treadmill $(n=40)$, significantly improved absolute claudication distance (ACD) by $40 \%$ above placebo levels [19]. We have not assessed the ability of TENS to improve ACD when used during daily life.

Although patient-centred education (SEDRIC) and TENS have both demonstrated potential to improve PA in people with $\mathrm{IC}$, the use of these components in combination has not previously been evaluated. Therefore, we do not know how potentially effective the combined intervention will be compared to each of the individual components. In addition, as part of the scaling process for complex intervention development, it is important to understand how the combined intervention can be feasibly delivered amongst patients with PAD and IC within the UK NHS. Equally key to informing the next stage of the project is the acceptability of the intervention as a whole or its individual components to patients with 
PAD and IC. Understanding these areas of uncertainty will address an important literature gap related to integrating two key components of pain management and patient education modalities in a patient-centred intervention to increase PA in individuals with PAD and IC.

To address these areas of uncertainty, we are undertaking a $2 \times 2$ factorial, assessor-blinded, parallel-group, placebo-controlled randomised trial of a Pain management and Patient Education for Physical Activity in Intermittent clauDication (PrEPAID) intervention. The aim of the trial is to determine the efficacy and feasibility of a TENS device used with or without a patient-centred education programme to improve walking distances in patients with PAD and IC. The following research questions will be addressed:

- What is the efficacy of the TENS device used with or without a patient-centred education programme to improve walking distances in patients with $\mathrm{PAD}$ and IC?

- What is the feasibility (i.e. recruitment and retention rates, adherence, safety, sample size for a definitive trial) of conducting a definitive randomised controlled trial (RCT) of TENS with and without patient-centred education in patients with PAD and IC?

- How acceptable are TENS and patient-centred education as interventions on their own or in combination to patients with IC?

\section{Methods/design}

Trial design

The PrEPAID trial is a $2 \times 2$ parallel group (TENS versus placebo TENS $\times$ patient education versus no additional education) feasibility RCT to compare the use of TENS against placebo TENS with and without a patient-centred education programme. Permuted block randomisation will be implemented to allocate patients to trial arms. The study design including patient inclusion, measurement and follow-up is shown in Fig. 1. Participants will be allocated to one of four groups ( $n=20$ per group): Active TENS, Active TENS + Patient education, Placebo TENS, Placebo TENS + Patient education. Active TENS/placebo and patient education interventions will be 6 weeks in duration, and all participants will receive secondary prevention therapy as recommended by NICE [11] including advice to exercise (usual care). Outcomes for all participants will be assessed at two points (at baseline and after 6 weeks of education programme and/or TENS (active or placebo). In addition, a subgroup of participants (those recruited in the first 8 months of recruitment) will be followed up 3 months post the 6-week intervention. All participants will be invited to attend a qualitative focus group to be conducted after final follow-up.

The study protocol has been developed based on the Standard Protocol Items: Recommendations for Interventional Trials (SPIRIT). The SPIRIT figure (Fig. 2) summarises the planned study conduct, review, reporting and interpretation. The completed SPIRIT checklist is presented in Additional file 1. The Consolidated Standards of Reporting Trials (CONSORT) extension to pilot and feasibility trials [20] and the Template for Interventional Description and Replication (TIDieR) guidelines [21] will be followed in reporting the final outcome of this trial.

\section{Trial settings}

This study is hosted at the Clinical Research Facility (CRF) of the Queen Elizabeth University Teaching Hospital, Glasgow, UK. Patients attending the NHS Greater Glasgow and Clyde vascular out-patient clinics will be invited to take part.

\section{Participant and eligibility criteria}

Patients (aged 40-85 years) with a history of stable IC and an ankle-brachial pressure index (ABPI) $\leq 0.9$ will be recruited. Participants would be included if they (1) have a clinical diagnosis of symptomatic PAD including resting ABPI $\leq 0.9$ in at least one leg; (2) have had stable IC for $\geq 3$ months; (3) have walking limited primarily by claudication; (4) are able to exercise on a treadmill; (5) are able to read and speak English to a level allowing satisfactory completion of the study procedures; (6) are able to provide written informed consent for participation. The following exclusion criteria will be applied to patients: (1) planned surgical or endovascular intervention for PAD within the next 3 months; (2) critical limb ischaemia; (3) the presence of any absolute contraindications to exercise testing/training as defined by the American College of Sports Medicine [22]; (4) previous experience of using TENS/ structured patient education for PAD; (5) contraindications to TENS (including epilepsy, dermatological conditions, indwelling electrical pumps/pacemakers); (6) inability to apply TENS independently (i.e. if a participant fails to demonstrate ability to apply TENS after receiving training); (7) patients who require walking aids including artificial limbs; (8) major surgery, myocardial infarction or stroke/transient ischaemic attack in the previous 6 months; (9) co-morbidities that cause pain or limit walking to a greater extent than IC (e.g. severe arthritis); (10) $>20 \%$ variation in baseline ACD on treadmill, taken at 2 weeks apart; (11) severe peripheral neuropathies above the ankle. 


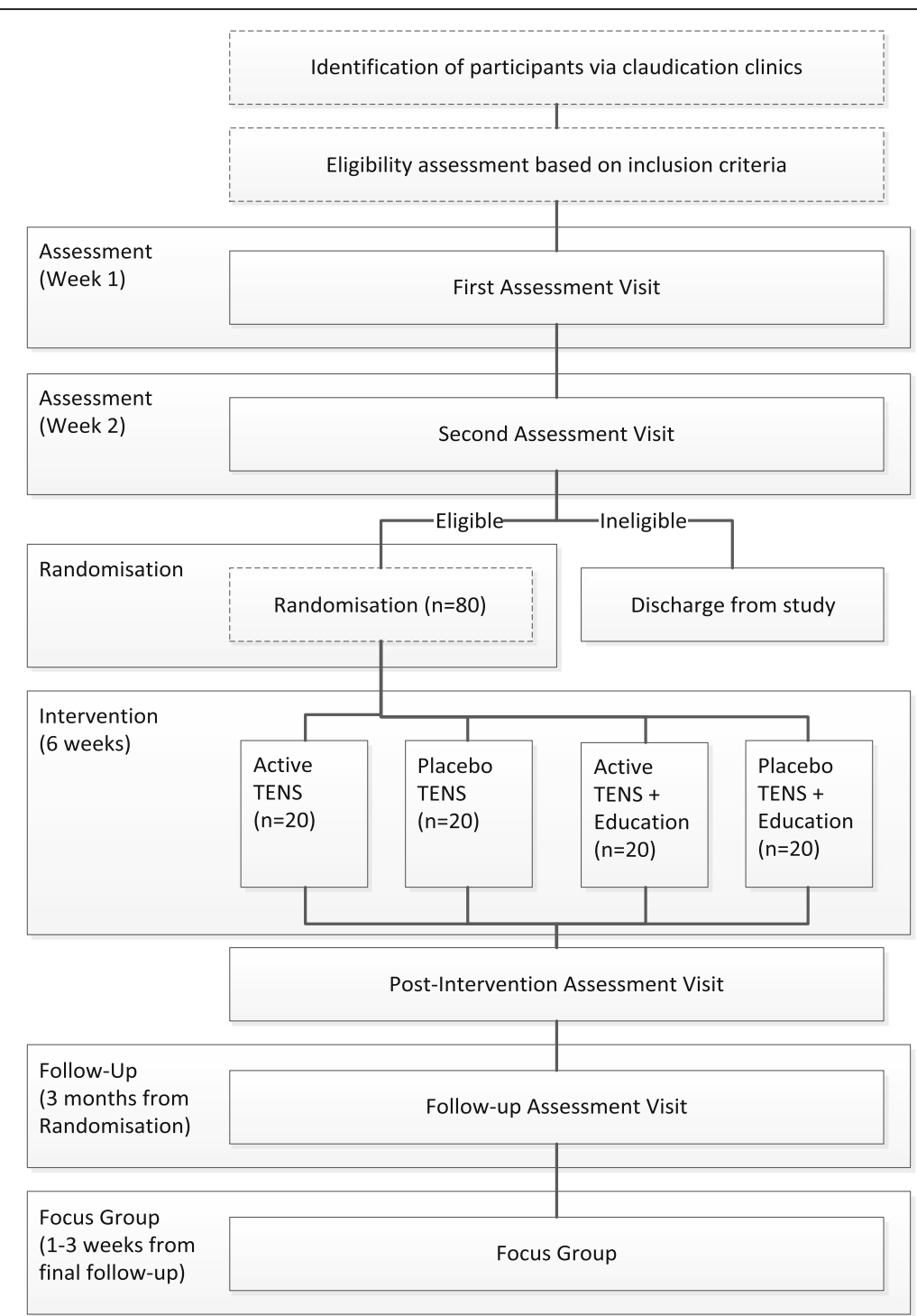

Fig. 1 Flowchart for participant identification, inclusion, study design, interventions, assessments and follow-up

\section{Identification of participants and consent}

Potential participants who are attending the vascular out-patient clinics within NHS Greater Glasgow and Clyde will be identified. They will be provided with a participant information sheet (Additional file 2), and contact details will be recorded on a study log. The nurse or other members of the study team will contact the patient, address any questions and arrange to meet. At this meeting, assuming the participant fulfils the trial eligibility criteria, informed consent (Additional file 3) may be taken by the nurse, or if the patient wishes, he/ she will be given more time to consider participating in the trial. Potential participants who have recently attended the claudication clinic will also be contacted by post and sent a brief outline of the study and the participant information sheet. They will be asked to return a prepaid response slip stating whether they wish to be contacted further regarding the study. If problems arise with recruitment, then the option of using the Safe Haven or primary care records to help identify patients diagnosed with PAD and IC will be explored and appropriate approval obtained.

\section{Randomisation}

Eligible and consented patients who have completed the baseline assessment and had $\leq 20 \%$ variation in ACD between the first and second visits will be randomly allocated to the trial arms. A central and independent randomisation facility (Internet-based randomisation system, the Interactive Web Response (IWR) system) will allocate the randomised therapy per patient. The IWR system, which will be based at the Robertson 


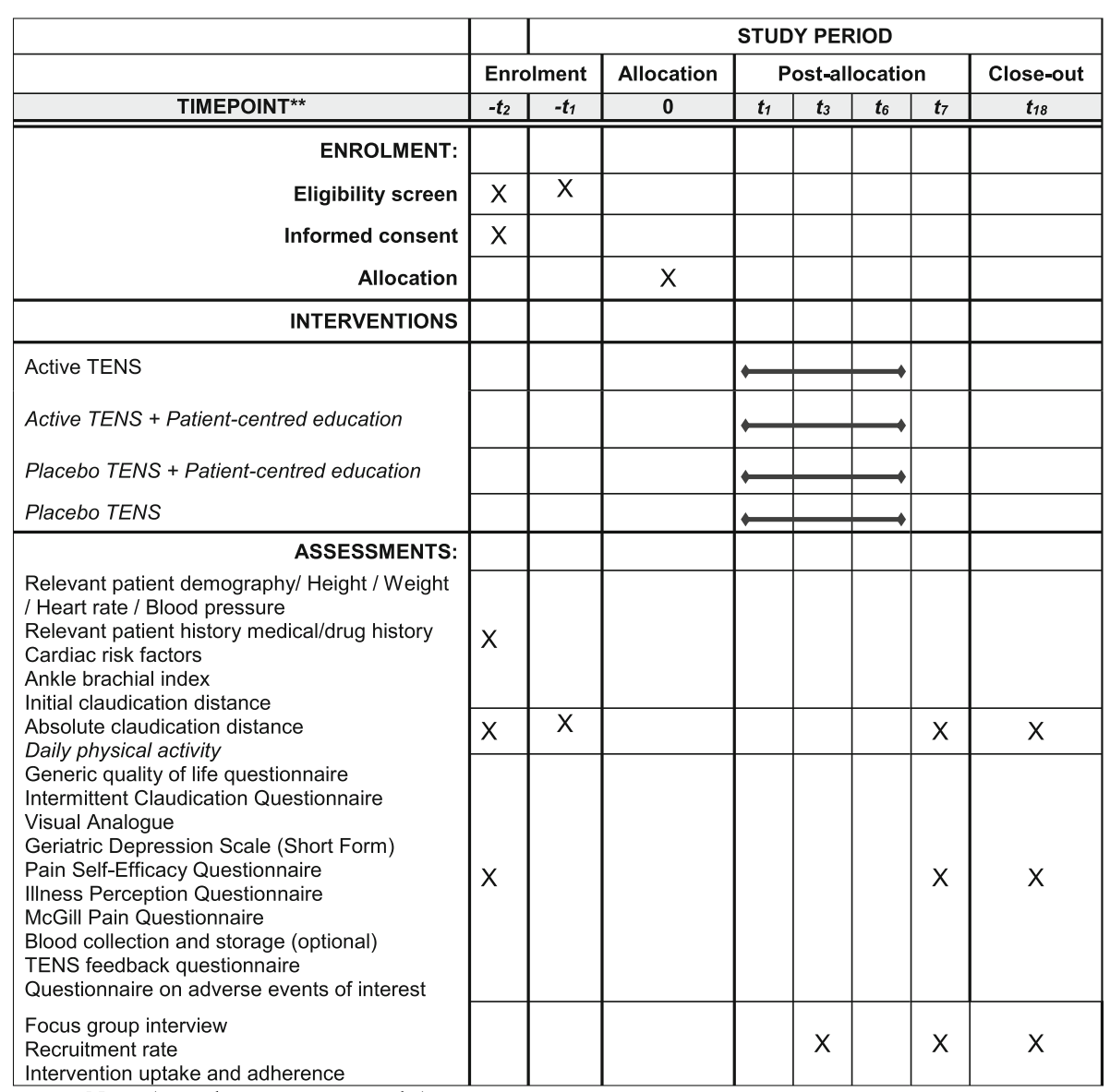

Note: (t numbers represent weeks)

Fig. 2 Standard Protocol Items: Recommendations for Interventional Trials (SPIRIT) figure (numbers beside $t$ represent weeks)

Institute University of Glasgow, will be available by web. A simple fixed block design (FBD) will be used to allocate patients to the groups. Randomisation outcome will be sent by email only to researchers involved in administering TENS and/or patient education. The outcome assessors and data analyst will be blinded after assignment to interventions.

\section{Sample size}

For the primary outcome measure, at $80 \%$ power and a two-tailed 5\% significance level, 16 participants per group will allow detection of an effect size of 1.0 standard deviation of ACD in the Active TENS group compared to the Placebo TENS control. Attrition rates in our previous pilot studies ranged from 7.1 [17] to $10 \%$ [19]. We will recruit 20 participants in each group, allowing for $20 \%$ attrition, and therefore aim to recruit 80 participants. If this effect size were applied to our separate pilot studies, this would provide the ability to detect a change of $169 \mathrm{~m}$ (TENS) [19] or $322 \mathrm{~m}$ (SEDRIC) [17] in our primary outcome measure of ACD. Indeed, in these studies, a sample size of 20 per group
(TENS) and 14 per intervention group (SEDRIC) was sufficient to detect a significant difference in this outcome measure.

\section{Intervention procedures Active TENS}

Participants will be given a TENS device at the intervention visit. They will be instructed to use the device daily at home or elsewhere. They will be specifically advised to use the device prior to or during a challenging walk each day. Challenging walks could be for activities of daily living or planned exercise. Those with bilateral claudication will be advised to wear the device on the worst limb and to alternate it as symptoms fluctuate. The Active TENS group will receive high-frequency TENS calibrated to $120 \mathrm{~Hz}, 200 \mu \mathrm{s}$, and will be free to set the intensity to a "strong but tolerable" level [19]. An MTR+ Dolito TENS machine (EME Services Ltd., Manchester, UK) will be used.

Treatment schedule Participants will be asked to wear the active TENS device every day as often as they can 
when they are awake and switch it on when they are standing/walking or about to engage in activity which they anticipate would trigger their IC pain. They will be instructed to switch it off after a maximum period of 1 $\mathrm{h}$, for a rest period of at least $10-20 \mathrm{~min}$, and to repeat this as often as warranted during daily activity.

Rationale for chosen TENS frequency In a proof-of-concept study, high-frequency TENS $(120 \mathrm{~Hz})$ was found to increase the distance IC patients walked before reaching pain tolerance, and that high-frequency TENS (compared to low-frequency TENS) was more effective at prolonging the time to reach pain threshold [19].

Attachment Patients will fit a TENS unit during wake periods and daily activity using two self-adhesive carbon rubber electrodes measuring $5 \times 5 \mathrm{~cm}$ (StiMus ${ }^{\bullet}$ Hydrogel Premium Self-Adhesive Electrodes, EME Services Ltd.) attached to the TENS unit via the manufacturer's leads. The area of pain reported by the participant would determine the electrode placement sites with the two electrodes to be placed at least $2 \mathrm{~cm}$ apart. Before it is provided to the patients for daily use, the TENS machine will be calibrated with a digital oscilloscope and tested manually by the research team.

\section{Placebo TENS}

Participants will receive the same model of TENS device and instructions for use as those in the active group except that the stimulation dose will be safely altered to produce non-therapeutic, ineffective stimulation $(6 \mathrm{~mA})$. This intensity setting will be locked off before the device is provided to the participants, and they will not be able to change it. This will allow the unit to be switched on with the appearance of a working unit. For the purposes of blinding, participants will be told that different dosages of TENS are being tested and for some of these dosages they might not feel anything even though the device is working. Indeed the placebo effect has been reported whilst using TENS in other conditions [23]; therefore, testing active TENS against placebo is recommended. All TENS units will be calibrated, checked and confirmed prior to being issued to participants. This method of achieving placebo has been successfully used in previous TENS trials [24-26].

\section{Patient-centred education}

The intervention for the groups receiving patient-centred education will be adopted from the successfully piloted SEDRIC study [17]: a one-off 3-h workshop of structured group education (four to five persons in each group) and three sets of twice-weekly phone calls. Two educators will implement the session. Training for educators will involve completion of the Diabetes Education and Self-Management for Ongoing and Newly Diagnosed (DESMOND) [27] core training; reading and demonstrating understanding of the SEDRIC [17] curriculum; and completion of at least two practice workshops that are quality assessed prior to delivering any sessions to patients. The aim of the structured education is to modify patients' illness beliefs and perceptions about IC by educating them on disease pathology and management philosophy. After the workshop, each patient will be supported to set goals for walking based around a pedometer (Yamax SW-200 Digi-Walker pedometers) and daily steps, and to develop an action plan regarding how these goals will be met. Participants will be encouraged to repeat this process for each new walking goal through twice-weekly phone calls from the educators during which the progress, barriers and challenges are further discussed, and new walking goals will be set.

\section{Trial schedule}

\section{Informed consent}

Written informed consent shall be obtained from each trial participant. The Research Nurse will explain the exact nature of the study in writing (by provision of the participant information sheet) and verbally, and will be responsible for consenting the participants. Trial participants will be informed that they are free to withdraw their consent from the study or study treatment at any time. Participants will be asked to attend the CRF at Queen Elizabeth University Hospital for a maximum of six occasions.

\section{Visit 1: first assessment visit}

At the first visit, participants will be assessed for eligibility, and those eligible will be requested to consent for further screening. Baseline outcome measurements will be conducted including weight/height/heart rate/blood pressure, ABPI, treadmill assessments, questionnaires and a blood sample. Participants will be fitted with the activPAL ${ }^{\text {ma }}$ monitor and advised to wear it continuously for 7 days. They will be given instructions on how to use the activPAL and provided with a sleep diary to complete during the duration of wearing the activPAL.

\section{Visit 2: second assessment visit (usually within 2 weeks after} visit 1)

Participants will then attend for a second visit to undergo a second treadmill test and to return the activPAL and sleep diary. Only participants with $\leq 20 \%$ variation in ACD will continue in the trial. After this visit, eligible participants will be randomised and given a date on which to return for intervention (TENS/Placebo \pm Patient education) as applicable. Recruitment and randomisation will be conducted in waves to allow groups to be formed for the education session. 
Visit 3: intervention visit (within 3 weeks after randomisation)

Participants attend the clinic to receive the TENS device and training instructions for its daily use as required, plus or minus the patient education per randomisation group. Participants allocated to receive education will undergo a structured group education session (four to six persons per group) according to the SEDRIC procedure [17]. Before the education session, participants allocated to receive TENS will be provided with the device and instructions for its use. The use of the device will also be demonstrated, and patients will be shown how to put it on and remove it. Participants will try out the device to be sure that they understand the procedure.

\section{Visit 4: post-intervention assessment visit (end of the 6- week intervention)}

The outcome assessments and procedure followed in visit 1 will be repeated including questionnaires, treadmill protocol, blood sample collection and fitting of activPAL. The treadmill test will be conducted by investigators who are blinded to the participants' group allocation. Participants will be given a prepaid envelope in which to return the activPAL monitor.

\section{Visit 5: follow-up assessment visit (3 months post- randomisation)}

All participants recruited within the first 8 months of recruitment will be invited to return for a 3 months follow-up visit. The outcome assessments and procedure followed in visits 1 and 4 will be repeated including questionnaires, treadmill protocol, blood sample collection and fitting of activPAL. The treadmill test will be conducted by investigators who are blinded to the participants' group allocation. Participants will be given a prepaid envelope in which to return the activPAL monitor. We shall allow assessment visit windows of \pm 2 weeks.

\section{Visit 6: focus group visit (usually 1-3 weeks after the final follow-up)}

All participants will be invited to participate in a focus group discussion. The discussion sessions, lasting $1 \mathrm{~h}$, will explore the acceptability of and satisfaction with the PrEPAID programme, components that were useful or not (in terms of helping them with PA) and participants' suggestions for changes. Each focus group will consist of four to six participants and will be facilitated by an independent investigator. The number of focus groups to be conducted will be determined by data saturation, and the sessions will be audio-recorded and transcribed verbatim.

\section{Laboratory tests}

At visits 1,4 and $5,20 \mathrm{ml}$ of blood will be taken from rested subjects. The samples will be spun and stored as per the standard operating procedure at the CRF at Queen Elizabeth University Hospital for future analysis of markers of angiogenesis and inflammatory response.

\section{Participant retention and withdrawal}

All reasonable efforts, within the CRF local standard operating procedure, will be made to ensure optimum participant engagement and to reduce study attrition. However, the study involves an intention-to-treat analysis; therefore, if a patient does not apply the TENS or attend the education class (if randomised to this arm), he/she will continue to be followed up. Nonetheless, all participants will have the right to withdraw from the study at any stage. If the participant is willing to provide them, the reasons for withdrawal will be documented and any data already collected from that participant will be analysed.

\section{Outcome definitions \\ Efficacy outcomes}

Measurements will be obtained at baseline, following the 6 -week intervention and at 3 months follow-up. The primary efficacy outcome will be the treadmill-assessed absolute claudication distance (ACD) (metres) using the Gardner treadmill protocol [28].

Secondary efficacy outcomes will include initial claudication distance (metres) assessed by a treadmill exercise using the Gardner treadmill protocol [28]. Daily PA will be assessed via activPAL data outcomes: total number of (1) steps; (2) upright events; (3) walking events; (4) event-based claudication index (ratio of walking events to upright events) participants undertake in a day [29]. Three days of activPAL data at each time point shall be specified as minimum for including a patient's activPAL data in the efficacy analysis.

Other secondary outcomes will assess patient-reported outcome measures. Disease-specific quality of life will be as assessed using the Intermittent Claudication Questionnaire [30]. Generic quality of life via the General Quality of Life Questionnaire (Short Form) [31], specifically the total item score as well as the two main scores (physical compound score and mental compound score), will be analysed. Pain intensity will assessed with the visual analogue scale, whilst pain quality will be recorded using the McGill Pain Questionnaire [32] $5 \mathrm{~min}$ after every treadmill test. Average pain intensity in the past 7 days will be recorded using the visual analogue scale [33]. Illness beliefs and psychosocial determinants of health and behaviour will be recorded using the Brief Illness Perception Questionnaire [34], the Geriatric Depression Scale: Short Form [35] and the Pain Self-Efficacy Questionnaire [36]. 


\section{Feasibility and acceptability outcomes}

Regarding recruitment rates, reasons for non-eligibility and non-recruitment of eligible patients will be recorded via the study screening log. Participants' retention throughout the trial and reasons for withdrawal will be documented. Adverse events in all groups will be monitored, recorded, managed and followed up. Intervention uptake (log of TENS use and attendance at education) and acceptability of these interventions will be measured via a questionnaire. TENS blinding fidelity will be assessed via a TENS feedback questionnaire. Outcome completion rate for all outcomes (number of days the activPAL is worn, treadmill test completion, patient-reported outcome measures at each outcome time point) will be assessed. Acceptability of the intervention will be assessed through focus group discussions at end of follow-up.

\section{Recording and reporting of adverse events}

We do not anticipate that the use of the CE-marked TENS device will result in any serious adverse events. Participants will be given prior information regarding the reporting of adverse events and measures to take including instructions to contact the research team via a dedicated phone line. At each study visit, participants will be specifically queried regarding the following adverse events of special interest: any case of itching, skin breakdown, mild electrical burn, other skin allergies or mild autonomic responses. Reported related adverse events will be documented in an applicable adverse event form.

\section{Timing of outcome assessments}

PA and patient-reported outcome measures will be assessed at baseline, at the end of the 6-week intervention (up to 2 weeks window) and 3 months post-randomisation (up to 2 weeks window). The recruitment, retention, outcome completion, intervention uptake and attrition rates will be assessed at the end of the study. Blinding and patients' acceptability of TENS and ease of use will be assessed at the point of patient exit from the study. Further assessment regarding patients' qualitative experience of the intervention will be conducted after 3 months post-randomisation (up to 2 weeks window).

\section{Statistics and data analysis}

The Robertson Centre for Biostatistics, part of the Glasgow Clinical Trials Unit, a fully registered UK Clinical Research Network (UKCRN) Clinical Trials Unit, will manage the trial data. Statistical analysis will be led by the study senior statistician (JG) at the Institute for Applied Health Research, Glasgow Caledonian University; the statistician is blinded to group allocation. Data analysis will be performed following a detailed prespecified statistical analysis plan, which will be published separately.
In summary, an intention-to treat analysis will be performed for the primary outcome on all randomised patients, except those who withdraw consent for the use of their data $[37,38]$. Baseline variables will be summarised using descriptive statistics. Also, the feasibility, acceptability, adverse events data and protocol and intervention adherence data will be summarised by randomised group and overall using descriptive statistics. Outcomes related to experience and perception via focus groups will be analysed by framework analysis $[39,40]$.

Comparisons will be undertaken to investigate the feasibility of studying the proposed outcomes for a definitive trial and to calculate estimates for the likely effect sizes and 95\% confidence intervals (CIs). To determine the feasibility of conducting a definitive trial, inferential analysis will be conducted at $95 \% \mathrm{CI}$, and the $p$ value will be set at $p<0.05$. The change in the primary outcome will be compared between and within groups using Mann-Whitney $U$ or Wilcoxon signed-rank tests (or their parametric equivalents) as applicable for between- and within-group comparisons. The log-rank method for pooled samples or substrata will be implemented where appropriate and when possible. Baseline participants' variability will be controlled for using the analysis of covariance. Also, other secondary efficacy analyses will examine differences in the changes in activPAL outcomes, initial claudication distance, patient-reported outcomes and effect scores calculated using Mann-Whitney $U$ or Wilcoxon signed-rank tests (or their parametric equivalents) as applicable for between- and within-group comparisons. Log-rank methods for pooled samples will be conducted where indicated. For the statistical analysis the software to be used is either SAS 9.2 for Windows (the SAS Institute, Cary, NC, USA) or SPSS Version 22.

\section{Data handling \\ Case report forms}

An electronic case report form (e-CRF) will be used to collect study data. The e-CRF will be developed by the study Data Centre at the Robertson Centre for Biostatistics, University of Glasgow, and access to the e-CRF will be restricted, with only authorised site-specific personnel able to make entries or amendments to the patients' data. It is the responsibility of the research team to ensure completion and to review and approve all data captured in the e-CRF.

All data handling procedures will be detailed in a study-specific data management plan. Data will be validated at the point of entry into the e-CRF and at regular intervals during the study. Data discrepancies will be flagged to the study site, and any data changes will be recorded in order to maintain a complete audit trail (reason for the change, date the change was made, who made the change). 


\section{Record retention}

To enable evaluations and/or audits from regulatory authorities, the investigators will keep records, including the identity of all participants (sufficient information to link records), all original signed informed consent forms, serious adverse event forms, source documents and detailed records of treatment disposition in accordance with the Good Clinical Practice guidelines, local regulations or as specified in the Clinical Study Agreement, whichever specifies a longer retention time. Data will be retained at the Data Centre for a minimum of 5 years.

\section{Trial management}

This research falls under the auspices of the clinical governance structure of Glasgow Caledonian University and NHS Greater Glasgow and Clyde CRF. The project is sponsored by Glasgow Caledonian University, and the Glasgow Caledonian University Research and Development Office will have responsibility for oversight, including audit of adherence to protocol and research governance standard operating procedures.

\section{Trial Management Group}

The trial will be coordinated from Glasgow Caledonian University by the Trial Management Group. This will consist of the co-investigators, NHS Greater Glasgow and Clyde CRF Research Nurse, the Robertson Centre for Biostatistics and the Glasgow Clinical Trial Unit. The Trial Management Group will be responsible for the overall management and completion of the project to timescales. The role of the group is to monitor all aspects of the conduct and progress of the trial, ensure that the protocol is adhered to and take appropriate action to safeguard participants and the quality of the trial itself. The group will meet bimonthly mainly via telephone conferences.

\section{Trial Steering Committee}

The Trial Steering Committee will utilise the strengths of diverse experts, including NHS Greater Glasgow and Clyde service users. This will help ensure that the research is relevant and accessible to a diverse audience. The committee will have an independent chair. Specifically, the committee will advise on the suitability of the interventions for the population group and design and participate in dissemination activities. The group members consist of the Chief Investigators (CS and JB), co-investigator (UA), two patient representatives, an expert in patient education interventions and PA behaviour change and an NHS management representative. The steering group will meet four times spread throughout the study with the aim of providing advice from a broad perspective.

\section{Protocol amendments}

Any change in the study protocol will require an amendment. Any proposed protocol amendments will be initiated by the Chief Investigators following discussion with the Trial Steering Committee, and any required amendment forms will be submitted to the ethics committee, funder, sponsor and NHS Greater Glasgow and Clyde Research and Development for approval as appropriate to their role. The Chief Investigators and the Trial Steering Committee will liaise with the study sponsor to determine whether an amendment is non-substantial or substantial. All amended versions of the protocol will be signed by the Chief Investigator and sponsor representative.

\section{Insurance and indemnity}

NHS indemnity is provided under the Clinical Negligence and Other Risks Indemnity Scheme and the Glasgow Caledonian University indemnity insurance.

\section{Study reports}

An annual progress report will be submitted to the funder, the Chief Science Office (CSO), Scotland, UK, the first being submitted 6 months from the date that all trial-related approvals are in place. Annual reports will be submitted to the ethics committee and sponsor, with the first submitted one year after the date that all trial-related approvals are in place. Recruitment data will, on a monthly basis, be uploaded to the UKCRN Portfolio database (and agreed successor to the database) through the mechanisms provided for the purpose, as part of the CSO requirement. Also, updated information on the outputs from the project shall be uploaded through the e-VAL system, which is now accessed through the ResearchFish website at https://researchfish. com/user/login?destination=awards. A final project report and other information and actions as required by the CSO as part of the project completion will be available and completed to the satisfaction of the CSO by the end of the funding period. Copies of all publications originating from this trial shall be provided to the CSO. The Chief Investigators and Project Management Group will produce all reports. All statistical reports will be produced by the Study Statistician from the Institute for Applied Health Research, Glasgow Caledonian University.

\section{Participants' data protection}

The data obtained from participants will remain confidential and stored securely at the Robertson Centre for Biostatistics University of Glasgow. The data are held in accordance with the Data Protection Act, which means that we keep it safely and cannot reveal it to other people without appropriate permission. The data held on the database will not be identifiable. In addition, PA 
data and basic demographic data will be kept on a password protected database on a secure server at Glasgow Caledonian University. The data held on the database will not be identifiable. This information collected may be used for further analysis by staff and students in the School of Health and Life Sciences at Glasgow Caledonian University at a later date.

\section{Trial dissemination}

The outcomes of the trials will be widely disseminated in journals and at scientific conferences.

\section{Discussion}

Individuals with peripheral arterial disease (PAD) and intermittent claudication (IC) are at high risk of cardiovascular events, hospitalisation and death [41-44]. Encouraging physical activity (PA) in individuals with IC is of both clinical and public health importance. Supervised exercise programmes are effective and are the recommended therapy to improve walking distances, cardiovascular fitness and quality of life benefits in individuals with IC. However, supervised exercise programmes are not readily available to most patients with PAD and IC, and, when available, the programmes record low patient engagement and high attrition.

In two recent systematic reviews, we identified patients' lack of disease understanding, uncertainty about exercise and the claudication pain as important barriers that prevent patients with PAD and IC from engaging in walking exercise [45], contributing to worse disease experience [46]. The results of these reviews underscored the importance of a self-management perspective to develop an intervention that concomitantly manages pain and educates patients about their disease pathology and the rationale of walking as a way of encouraging patients to adopt and adhere to the walking exercise recommendation. We also identified important intervention components through a series of systematic reviews, and we have conducted a series of laboratory, proof-of-concept and pilot studies underpinning both components of PrEPAID. We subsequently developed PrEPAID following the Medical Research Council (MRC) framework for complex interventions [47].

Whilst patient-centred education and TENs for pain management have both individually demonstrated potential to increase PA in individuals with PAD and IC, the use of these components together has not been evaluated. In line with the MRC framework for complex intervention development and evaluation, this trial will demonstrate the feasibility and potential benefit of TENS used with or without patient-centred education to improve PA in individuals with PAD and IC. This is an important consideration, since the two components of the intervention primarily target different important barriers to walking in this population. Findings will also be important in refining and tailoring the next phase of the intervention as well as in estimating sample size for the full RCT.

\section{Trial status}

The study is ongoing at the time of submitting this manuscript (December 2018). This trial was using protocol version 2.0 (12 September 2017) at the time of this submission. Recruitment started in the Queen Elizabeth University Hospital on 17 May 2018 and is expected to be completed on 17 July 2019. The trial management committee manages and disseminates the protocol amendments.

\section{Additional files}

Additional file 1: Completed SPIRIT checklist for PrEPAID trial. (DOC $382 \mathrm{~kb}$ ) Additional file 2: Participant information sheet for PrEPAID trial. (DOCX $190 \mathrm{~kb}$ )

Additional file 3: Participant consent form for PrEPAID trial. (DOCX 217 kb)

\section{Abbreviations}

ABPI: Ankle-brachial pressure index; ACD: Absolute claudication distance; CRF: Clinical Research Facility; CSO: Chief Science Office; DESMOND: Diabetes Education and Self-Management for Ongoing and Newly Diagnosed; IC: Intermittent claudication; NHS: National Health Service; NICE: National Institute for Health and Care Excellence; PA: Physical activity; PAD: Peripheral arterial disease; PrEPAID: Pain management and Patient Education for Physical Activity in Intermittent clauDication; RCT: Randomised controlled trial; SEDRIC: Structured EDucation for Rehabilitation in Intermittent Claudication; TENS: Transcutaneous electrical nerve stimulation

\section{Acknowledgements}

Liam Humphreys, Helen Crank, Catherine Hewitt, Shah Nawaz, Wissam Al-Jundi, Hazel Trender, Jonathan Michaels and Trish Gorely were part of the group who conceptualised and developed SEDRIC, on which the education component of PrEPAID is based.

\section{Trial sponsor}

Glasgow Caledonian University is the trial sponsor (sponsor's Protocol Number 16-044). The sponsor representative is Professor James Woodburn, Associate Dean Research/Director of Centre for Living School of Health and Life Sciences Glasgow Caledonian University (email

Jim.Woodburn@gcu.ac.uk).

\section{Funding}

The trial is funded by Chief Scientist Office (CSO) Scotland Translational grant award (TCS/16/55). The first author (UA) is being sponsored by the University of Nigeria through the TETFUND for a PhD being conducted at Glasgow Caledonian University. This protocol is part of a work towards the award of his PhD. The funders have no role in the study design, collection, management, analysis or interpretation of data; writing of this report; or decision to submit this report for publication.

Availability of data and materials

An anonymous copy of the final datasets underlying publications resulting from this trial will be shared upon reasonable and approved request. Request may be made through email to the Principal Investigator and can only be made upon meeting the terms and conditions for the ethics approval of this trial. 


\section{Authors' contributions}

UA conceived and led the study design. JB, PD, GT, WS, JG and CS contributed to the design of the study. UA drafted the manuscript. All authors contributed to manuscript revision and read and approved the final manuscript.

\section{Ethics approval and consent to participate}

The study protocol was approved by the West of Scotland Research Ethic Committee 4 (17/WS/0094) and the NHS Greater Glasgow and Clyde Clinical Research and Development (GN16CE378). Written informed consent shall be obtained from each trial participant. The Research Nurse or another member of the study team will explain the exact nature of the study in writing and verbally, will provide the participant information sheets and will be responsible for consenting the participants. Trial participants will be informed that they are free to withdraw their consent from the study or study treatment at any time.

\section{Consent for publication}

Not applicable.

\section{Competing interests}

The authors declare that they have no competing interests.

\section{Publisher's Note}

Springer Nature remains neutral with regard to jurisdictional claims in published maps and institutional affiliations.

\begin{abstract}
Author details
${ }^{1}$ Centre for Living, School of Health and Life Sciences, Glasgow Caledonian University, Glasgow, UK. ${ }^{2}$ Department of Medical Rehabilitation, Faculty of Health Science and Technology, College of Medicine, University of Nigeria, Enugu, Nigeria. ${ }^{3}$ Department of Physiotherapy and Paramedicine, School of Health and Life Sciences, Glasgow Caledonain University, Glasgow, UK. ${ }^{4}$ Vascular Surgery NHS Greater Glasgow and Clyde Health Board, Glasgow, UK. ${ }^{5}$ Institute of Cardiovascular and Medical Sciences, University of Glasgow, Glasgow, UK. ${ }^{6}$ Department of Sport, Exercise and Rehabilitation, Northumbria University, Newcastle, UK. Institute of Applied Health Research, Glasgow Caledonian University, Glasgow, UK.
\end{abstract}

Received: 7 January 2019 Accepted: 19 March 2019

Published online: 16 April 2019

\section{References}

1. Hankey GJ, Norman PE, Eikelboom JW. Medical treatment of peripheral arterial disease. JAMA. 2006;295:547-53.

2. Olin JW, Sealove BA. Peripheral artery disease: current insight into the disease and its diagnosis and management. Mayo Clin Proc. 2010;85:678-92.

3. Serrano Hernando FJ, Martín Conejero A. Peripheral artery disease: pathophysiology, diagnosis and treatment. Rev Española Cardiol. 2007;60: 969-82.

4. Muir RL. Peripheral arterial disease: pathophysiology, risk factors, diagnosis, treatment, and prevention. J Vasc Nurs. 2009;27:26-30.

5. Hamburg NM, Creager MA. Pathophysiology of intermittent claudication in peripheral artery disease. Circ J. 2017;81:281-9.

6. McDermott MMG. Lower extremity manifestations of peripheral artery disease: the pathophysiologic and functional implications of leg ischemia. Circ Res. 2015;116:1540-50.

7. Regensteiner JG, Hiatt WR, Coll JR, Criqui MH, Treat-Jacobson D, McDermott $\mathrm{MM}$, et al. The impact of peripheral arterial disease on health-related quality of life in the Peripheral Arterial Disease Awareness, Risk, and Treatment: New Resources for Survival (PARTNERS) Program. Vasc Med. 2008;13:15-24.

8. Breek JC, De Vries J, Van Heck GL, Van Berge Henegouwen DP, Hamming JF. Assessment of disease impact in patients with intermittent claudication: discrepancy between health status and quality of life. J Vasc Surg. 2005:41:443-50.

9. Gerhard-Herman MD, Gornik HL, Barrett C, Barshes NR, Corriere MA, Drachman DE, et al. AHA ACC guideline on the management of patients with lower extremity peripheral artery disease: a report of the American College of Cardiology/American Heart Association Task Force on Clinical Practice Guidelines. Circulation. 2016;2017:e726-79.
10. Garg PK, Tian L, Criqui MH, Liu K, Ferrucci L, Guralnik JM, et al. Physical activity during daily life and mortality in patients with peripheral arterial disease. Circulation. 2006;114:242-8.

11. Layden J, Michaels J, Bermingham S, Higgins B. Diagnosis and management of lower limb peripheral arterial disease: summary of NICE guidance. BMJ. 2012;345:e4947.

12. Fokkenrood $\mathrm{H}$, Bendermacher $\mathrm{B}$, Lauret $\mathrm{G}$, Willigendael $\mathrm{E}$, Prins $\mathrm{M}$, Teijink J. Supervised exercise therapy versus non-supervised exercise therapy for intermittent claudication. Cochrane Database Syst Rev. 2013;8:CD005263.

13. Shalhoub J, Hamish M, Davies AH. Supervised exercise for intermittent claudication - an under-utilised tool. Ann R Coll Surg Engl. 2009;91:473-6.

14. Gorely T, Crank H, Humphreys L, Nawaz S, Tew GA. "Standing still in the street": experiences, knowledge and beliefs of patients with intermittent claudication-a qualitative study. J Vasc Nurs. 2015;33:4-9.

15. Galea MN, Bray SR, Martin Ginis KA. Barriers and facilitators for walking in individuals with intermittent claudication. J Aging Phys Act. 2008;16:69-84.

16. Abaraogu UO, Dall PM, Seenan CA. The effect of structured patient education on physical activity in patients with peripheral arterial disease and intermittent claudication: a systematic review. Eur J Vasc Endovasc Surg. 2017;54:58-68.

17. Tew GA, Humphreys L, Crank H, Hewitt C, Nawaz S, Al-Jundi W, et al. The development and pilot randomised controlled trial of a group education programme for promoting walking in people with intermittent claudication. Vasc Med. 2015;20:348-57.

18. Seenan C, Roche PA, Tan C-W, Mercer T. Modification of experimental, lower limb ischemic pain with transcutaneous electrical nerve stimulation. Clin J Pain. 2012;28:693-9.

19. Seenan C, McSwiggan S, Roche PA, Tan C-W, Mercer T, Belch JJF. Transcutaneous electrical nerve stimulation improves walking performance in patients with intermittent claudication. J Cardiovasc Nurs. 2016;31:323-30

20. Eldridge SM, Chan CL, Campbell MJ, Bond CM, Hopewell S, Thabane L, et al. CONSORT 2010 statement: Extension to randomised pilot and feasibility trials. BMJ. 2016:355:15239.

21. Hoffmann TC, Glasziou PP, Boutron I, Milne R, Perera R, Moher D, et al. Better reporting of interventions: template for intervention description and replication (TIDieR) checklist and guide. BMJ. 2014;348:g1687.

22. Thompson PD, Arena R, Riebe D, Pescatello LS. American College of Sports Medicine. ACSM's New Preparticipation Health Screening Recommendations from ACSM's Guidelines for Exercise Testing and Prescription (9th Ninth Edition). Curr Sports Med Rep. 2013;12(4):215-7.

23. Vance CGT, Dailey DL, Rakel BA, Sluka KA. Using TENS for pain control: the state of the evidence. Pain Manag. 2014:4:197-209.

24. Ryan CG, King R, Robinson V, Punt TD, Dinse HR, Grunenberg C, et al. Transcutaneous electrical nerve stimulation using an LTP-like repetitive stimulation protocol for patients with upper limb complex regional pain syndrome: a feasibility study. Hand Therapy. 2016;0:1-12.

25. Francis RP, Marchant $P$, Johnson Ml. Conventional versus acupuncture-like transcutaneous electrical nerve stimulation on cold-induced pain in healthy human participants: effects during stimulation. Clin Physiol Funct Imaging. 2011:31:363-70.

26. Ryan CG, King R, Robinson V, Punt TD, Dinse HR, Grunenberg C, et al. Transcutaneous electrical nerve stimulation using an LTP-like repetitive stimulation protocol for patients with upper limb complex regional pain syndrome: a feasibility study. Hand Ther. 2017;22:52-63.

27. Skinner TC, Carey ME, Cradock S, Daly H, Davies MJ, Doherty Y, et al. Diabetes education and self-management for ongoing and newly diagnosed (DESMOND): process modelling of pilot study. Patient Educ Couns. 2006;64:369-77.

28. Gardner AW, Skinner JS, Cantwell BW, Smith LK. Progressive vs single-stage treadmill tests for evaluation of claudication. Med Sci Sports Exerc. 1991;23:402-8.

29. Clarke CL, Holdsworth RJ, Ryan CG, Granat MH. Free-living physical activity as a novel outcome measure in patients with intermittent claudication. Eur J Vasc Endovasc Surg. 2013;45:162-7.

30. Chong PFS, Garratt AM, Golledge J, Greenhalgh RM, Davies AH. The Intermittent Claudication Questionnaire: a patient-assessed conditionspecific health outcome measure. J Vasc Surg. 2002;36:764-71.

31. Ware JE. SF-36 ${ }^{\circledR}$ Health Survey Update [Internet]. SF Community. 2010. p. 1-16.

32. Melzack R. The McGill Pain Questionnaire. Anesthesiology. 2005;103:199-202.

33. Huskisson EC. Measurement of pain. Lancet. 1974;304:1127-31. 
34. Broadbent E, Petrie KJ, Main J, Weinman J. The Brief Illness Perception Questionnaire. J Psychosom Res. 2006;60:631-7.

35. Greenberg SA. How to try this: the Geriatric Depression Scale: Short Form. Am J Nurs. 2007:107:60-9.

36. Nicholas MK. The Pain Self-Efficacy Questionnaire: taking pain into account. Eur J Pain. 2007;11:153-63.

37. Fergusson D. Post-randomisation exclusions: the intention to treat principle and excluding patients from analysis. BMJ. 2002;325:652-4.

38. McCoy E. Understanding the intention-to-treat principle in randomized controlled trials. West J Emerg Med. 2017;18:1075-8.

39. Gale NK, Heath G, Cameron E, Rashid S, Redwood S. Using the framework method for the analysis of qualitative data in multi-disciplinary health research. BMC Med Res Methodol. 2013;13:117.

40. Parkinson S, Eatough V, Holmes J, Stapley E, Midgley N. Framework analysis: a worked example of a study exploring young people's experiences of depression. Qual Res Psychol. 2016;13:109-29.

41. Sigvant B, Lundin F, Wahlberg E. The risk of disease progression in peripheral arterial disease is higher than expected: a meta-analysis of mortality and disease progression in peripheral arterial disease. Eur. J. Vasc. Endovasc. Surg. 2016;51:395-403.

42. Rantner B, Kollerits B, Pohlhammer J, Stadler M, Lamina C, Peric S, et al. The fate of patients with intermittent claudication in the 21st century revisited — results from the CAVASIC Study. Sci Rep. 2017:8:45833.

43. Cambou JP, Aboyans V, Constans J, Lacroix P, Dentans C, Bura A. Characteristics and outcome of patients hospitalised for lower extremity peripheral artery disease in France: the COPART Registry. Eur J Vasc Endovasc Surg. 2010;39:577-85.

44. Vaartjes I, de Borst GJ, Reitsma JB, de Bruin A, Moll FL, Grobbee DE, et al. Longterm survival after initial hospital admission for peripheral arterial disease in the lower extremities. BMC Cardiovasc Disord. 2009;9:43.

45. Abaraogu U, Ezenwankwo E, Dall P, Tew G, Stuart W, Brittenden J, et al. Barriers and enablers to walking in individuals with intermittent claudication: a systematic review to conceptualize a relevant and patient-centered program. PLOS One. 2018;13:e0201095.

46. Abaraogu UO, Ezenwankwo EF, Dall PM, Seenan CA. Living a burdensome and demanding life: a qualitative systematic review of the patients experiences of peripheral arterial disease. PLOS One. 2018;13:e0207456.

47. Craig P, Dieppe P, Macintyre S, Michie S, Nazareth I, Petticrew M. Developing and evaluating complex interventions: the new Medical Research Council guidance. BMJ. 2008;337:a1655.

Ready to submit your research? Choose BMC and benefit from:

- fast, convenient online submission

- thorough peer review by experienced researchers in your field

- rapid publication on acceptance

- support for research data, including large and complex data types

- gold Open Access which fosters wider collaboration and increased citations

- maximum visibility for your research: over $100 \mathrm{M}$ website views per year

At $\mathrm{BMC}$, research is always in progress.

Learn more biomedcentral.com/submissions 